\title{
Perfect codes in Cayley sum graphs
}

\author{
Xuanlong $\mathrm{Ma}^{*}$ \\ School of Science \\ Xi'an Shiyou University \\ Xi'an 710065, China \\ xuanlma@xsyu.edu.cn \\ Kaishun Wang ${ }^{\dagger}$
}

Laboratory of Mathematics and Complex Systems (MOE)

School of Mathematical Sciences, Beijing Normal University

Beijing 100875, China

wangks@bnu .edu.cn

Yuefeng Yang $\ddagger$

School of Science

China University of Geosciences

Beijing 100083, China

yangyf@cugb.edu.cn

Submitted: Aug 19, 2020; Accepted: Dec 6, 2021; Published: Jan 28, 2022

(C) The authors. Released under the CC BY-ND license (International 4.0).

\begin{abstract}
A subset $C$ of the vertex set of a graph $\Gamma$ is called a perfect code of $\Gamma$ if every vertex of $\Gamma$ is at distance no more than one to exactly one vertex in $C$. Let $A$ be a finite abelian group and $T$ a square-free subset of $A$. The Cayley sum graph of $A$ with respect to the connection set $T$ is a simple graph with $A$ as its vertex set, and two vertices $x$ and $y$ are adjacent whenever $x+y \in T$. A subgroup of $A$ is said to be a subgroup perfect code of $A$ if the subgroup is a perfect code of some Cayley sum graph of $A$. In this paper, we give some necessary and sufficient conditions for a subset of $A$ to be a perfect code of a given Cayley sum graph of $A$. We also characterize all subgroup perfect codes of $A$.
\end{abstract}

Mathematics Subject Classifications: 05C25, 05C69, 94B25

*Supported by NSFC grant 11801441 and 61976244, the Natural Science Basic Research Program of Shaanxi grant 2020JQ-761, and the Young Talent fund of University Association for Science and Technology in Shaanxi grant 20190507.

${ }^{\dagger}$ Supported by the National Key R\&D Program of China grant 2020YFA0712900, and NSFC grant 12071039 and 12131011.

${ }^{\ddagger}$ Corresponding author. Supported by NSFC grant 12101575, and the Fundamental Research Funds for the Central Universities grant 2652019319. 


\section{Introduction}

In this paper, every group considered is finite, and every graph considered is finite and simple. For a graph $\Gamma$ with vertex set $V$, a subset $C$ of $V$ is called a perfect code [16] of $\Gamma$ if every vertex of $\Gamma$ is at distance no more than one to exactly one vertex in $C$. In other words, $C$ is a perfect code in $\Gamma$ provided that $C$ is independent in $\Gamma$ and every vertex of $V \backslash C$ is adjacent to precisely one vertex of $C$. In some references, a perfect code is also called an efficient dominating set [9, 8] or independent perfect dominating set [17]. Since the beginning of coding theory in the late 1940s, perfect codes have been one of the most important objects of study in information theory. See, for example, the two surveys $[13,23]$ on perfect codes and related definitions in the classical setting. Since the seminal paper of Biggs [2] and the fundamental work of Delsarte [7], perfect codes in distancetransitive graphs and, in general, in distance-regular graphs and association schemes have received considerable attention in the literature. Beginning with [16], a great amount of work on perfect codes in general graphs has been produced. See, for example, [6, 22, 27].

In the past a few years, perfect codes in Cayley graphs have attracted considerable attention, see, for example, [11, 28, 29]. In [14], Huang, Xia and Zhou first introduced the concept of a perfect code of a group $G$. A subset $C$ of $G$ is said to be a perfect code of $G$ if $C$ is a perfect code of some Cayley graph of $G$. In particular, a subgroup is said to be a subgroup perfect code of $G$ if the subgroup is also a perfect code of $G$. Also in [14], they gave a necessary and sufficient condition for a normal subgroup of a group $G$ to be a subgroup perfect code of $G$, and determined all the subgroup perfect codes of dihedral groups and some abelian groups. For more results on subgroup perfect codes of Cayley graphs, see [3, 20, 25, 26].

In 1989, Chung [5] first introduced the concept of a Cayley sum graph of an abelian group. Let $A$ be an abelian group. Given a subset $T$ of $A$, the Cayley sum graph (also called addition Cayley graph) of $A$ with respect to the connection set $T$, denoted by $\operatorname{CayS}(A, T)$, is a graph with $A$ as its vertex set, and two elements $x$ and $y$ are adjacent whenever $x+y \in T$. An element $x$ of $A$ is said to be a square if $x=2 y$ for some $y \in A$. A subset of $A$ without squares is called a square-free subset of $A$. Since every graph considered in this paper is simple, we always consider a simple Cayley sum graph $\operatorname{CayS}(A, T)$ of an abelian group $A$, that is, the connection set $T$ should be square-free. More explicitly, for a square-free subset $T$ of $A$, the Cayley sum graph $\operatorname{CayS}(A, T)$ of $A$ with respect to the connection set $T$ is a simple graph with $A$ as its vertex set, and two elements $x$ and $y$ are adjacent whenever $x+y \in T$. One can easily verify that $\operatorname{CayS}(A, T)$ is $|T|$-regular. In [12], Grynkiewicz, Levb and Serra pointed out that, as the twins of the usual Cayley graphs, Cayley sum graphs are rather difficult to study, so they received much less attention in the literature. For most results about Cayley sum graphs, see $[1,4,18,15,10,21]$.

Huang, Xia and Zhou [14] first introduced the definition of a subgroup perfect code of a group $G$, that is a subgroup of $G$ and a perfect code of a Cayley graph of $G$. In [19], the first author, Feng and the second author studied the perfect codes of CayS $(A, T)$, and defined a subgroup perfect code of an abelian group by using Cayley sum graphs instead 
of Cayley graphs. More precisely, a subgroup of $A$ is said to be a subgroup perfect code of $A$ if the subgroup is a perfect code of some Cayley sum graph of $A$. Also, in [19], the authors reduced the problem of determining when a given subgroup of an abelian group is a perfect code to the case of abelian 2-groups, and classified the abelian groups whose all non-trivial subgroups are perfect codes.

In this paper, we study perfect codes of a simple Cayley sum graph of an abelian group. For any abelian group $D$ of odd order, since $\{2 g: g \in D\}=D$, it follows that every element of $D$ is a square. Therefore, the only simple Cayley sum graph of an abelian group $D$ of odd order is the empty Cayley sum graph $\operatorname{CayS}(D, \varnothing)$. To avoid this kind of triviality, we only consider abelian groups of even order. In other words, throughout this paper, $A$ denotes a finite abelian group of even order, and the identity element of $A$ is denoted by 0 .

The remainder of this paper is organized as follows. In Section 2, we give some necessary and sufficient conditions for a subset of $A$ to be a perfect code of a given Cayley sum graph of $A$ (see Theorem 4 and Corollary 6). In Section 3, we determine the structure of a subgroup perfect code of $A$ (see Theorem 10), which improves [19, Theorem 3.1], and we also give some applications of Theorem 10 (see Propositions 18, 19 and 20).

\section{$2 \quad$ Perfect codes}

In this section, we study perfect codes of a Cayley sum graph of $A$ and give some necessary and sufficient conditions for a subset of $A$ to be a perfect code of a given Cayley sum graph of $A$ (see Theorem 4 and Corollary 6 ).

For two subsets $B$ and $C$ of $A$, we write

$$
B \pm C=\{b \pm c: b \in B, c \in C\},
$$

which is abbreviated by $b \pm C$ in the case where $B=\{b\}$.

Lemma 1. Take a subset $X$ of vertices in a Cayley sum graph CayS $(A, T)$. Then every element of $A \backslash X$ is adjacent to at least one vertex of $X$ in $\operatorname{CayS}(A, T)$ if and only if

$$
A \backslash X \subseteq \bigcup_{t \in T}(t-X)
$$

Proof. Observe that $\bigcup_{t \in T}(t-X)$ consists of all vertices that are adjacent to some vertices of $X$. Hence, the desired result follows.

Lemma 2. Take a subset $X$ of vertices in a Cayley sum graph $\operatorname{CayS}(A, T)$. Then the following are equivalent:

(i) Every element of $A$ is adjacent to at most one element of $X$ in $\operatorname{CayS}(A, T)$;

(ii) For each two distinct elements $t_{1}$ and $t_{2}$ in $T$, we have $\left(t_{1}-X\right) \cap\left(t_{2}-X\right)=\varnothing$;

(iii) $(X-X) \cap(T-T)=\{0\}$. 
Proof. Suppose that (i) holds. Assume that $\left(t_{1}-X\right) \cap\left(t_{2}-X\right) \neq \varnothing$ for some elements $t_{1}$ and $t_{2}$ in $T$. Then $t_{1}-x_{1}=t_{2}-x_{2}$ for some elements $x_{1}$ and $x_{2}$ in $X$. The fact that $T$ is square-free indicates that $t_{1}-x_{1} \neq x_{1}$ and $t_{1}-x_{1}=t_{2}-x_{2} \neq x_{2}$. It follows that $t_{1}-x_{1}$ is adjacent to both $x_{1}$ and $x_{2}$, which implies that $x_{1}=x_{2}$, and so $t_{1}=t_{2}$. Thus, (ii) is valid.

It is clear that (ii) implies (iii). Now suppose that (iii) holds. We only need to prove (i). Assume for a contrary that there exists an element $y$ in $A$ which is adjacent to distinct elements $y_{1}$ and $y_{2}$ of $X$. Then $y+y_{1} \in T$ and $y+y_{2} \in T$, and so $y_{1}-y_{2} \in(X-X) \cap(T-T)$, a contradiction. This completes the proof.

Lemma 3. Take a subset $X$ of vertices in a Cayley sum graph $\operatorname{CayS}(A, T)$. Then the following are equivalent:

(i) $X$ is an independent set of $\operatorname{CayS}(A, T)$;

(ii) For each $t \in T$, we have $X \cap(t-X)=\varnothing$;

(iii) $(X+X) \cap T=\varnothing$.

Proof. Now assume that (i) holds. Suppose to the contrary that there exists $t \in T$ such that $X \cap(t-X) \neq \varnothing$. Then there exist $x_{1}, x_{2} \in X$ such that $x_{1}=t-x_{2}$. Since $t$ is not a square, we have $x_{1} \neq x_{2}$. It follows that $x_{1}$ is adjacent to $x_{2}$, this contradicts the fact that $X$ is an independent set. Thus, (ii) is valid.

Also, it is easy to see that (ii) implies (iii), and (iii) implies (i). Thus, the proof is complete.

Theorem 4. For a Cayley sum graph $\operatorname{CayS}(A, T)$, write $T=\left\{t_{1}, \ldots, t_{s}\right\}$ and take a subset $X$ of $A$. The following are equivalent:

(i) $X$ is a perfect code of $\operatorname{CayS}(A, T)$;

(ii) $\left\{X, t_{1}-X, \ldots, t_{s}-X\right\}$ is a partition of $A$;

(iii) $|A|=|X|(s+1),(X+X) \cap T=\varnothing$ and $(X-X) \cap(T-T)=\{0\}$.

Proof. We first prove (i) implies (iii). Suppose that (i) holds. By the definition of a perfect code, we have $|A|=|X|(s+1)$. Also, in view of Lemmas 2 and 3, we conclude that (iii) holds. Now by Lemmas 2 and 3 again, it follows that (iii) implies (ii).

Now suppose that (ii) holds. It suffices to prove (i). Note that

$$
A=X \dot{\cup}\left(t_{1}-X\right) \dot{U} \cdots \dot{\cup}\left(t_{s}-X\right) \text {. }
$$

Then $A \backslash X=\bigcup_{t \in T}(t-X)$. By Lemma 1, we have that every element of $A \backslash X$ is adjacent to at least one vertex of $X$ in $\operatorname{CayS}(A, T)$. Moreover, (1) implies that $\left(t_{1}-X\right) \cap\left(t_{2}-X\right)=\varnothing$ for each two distinct elements $t_{1}$ and $t_{2}$ in $T$. It follows from Lemma 2 that every vertex in $A \backslash X$ is adjacent to exactly one vertex in $X$ in $\operatorname{CayS}(A, T)$. Notice that (1) also implies that $X \cap(t-X)=\varnothing$ for each $t \in T$. According to Lemma $3, X$ is an independent set of $\operatorname{CayS}(A, T)$, and so $X$ is a perfect code of CayS $(A, T)$, as desired. 
Two subsets $M$ and $N$ of $A$ are supplementary, denoted by $A=M \oplus N$, if each element $a$ of $A$ can be written in a unique way as $m+n$, where $m \in M$ and $n \in N$.

Lemma 5. ([24, Proposition 2.1]) Let $M$ and $N$ be two subsets of $A$. Then $A=M \oplus N$ is equivalent to the conjunction of any two of the following conditions:

(a) $A=M+N$;

(b) $(M-M) \cap(N-N)=\{0\}$;

(c) $|A|=|M||N|$.

Corollary 6. For a Cayley sum graph $\operatorname{CayS}(A, T)$, take an inverse-closed subset $X$ in $A$. Then $X$ is a perfect code of CayS $(A, T)$ if and only if $A=X \oplus T^{0}$, where $T^{0}=T \cup\{0\}$.

Proof. Since $T$ is square-free, one obtains $0 \notin T$, which implies that $\left|T^{0}\right|=|T|+1$.

We first prove the sufficiency. Suppose that $A=X \oplus T^{0}$. Then by Lemma 5, we have $|A|=|X|(|T|+1)$ and $(X-X) \cap\left(T^{0}-T^{0}\right)=\{0\}$. Since $T-T \subseteq T^{0}-T^{0}$ and $T \subseteq T^{0}-T^{0}$, one gets $(X-X) \cap(T-T)=\{0\}$ and $(X-X) \cap T=\varnothing$. The fact that $X$ is inverse-closed implies that $(X+X) \cap T=\varnothing$. It follows from Theorem 4 that $X$ is a perfect code of CayS $(A, T)$, as desired.

We next prove the necessity. Suppose that $X$ is a perfect code of CayS $(A, T)$. By Theorem 4, we have $|A|=|X|(|T|+1),(X+X) \cap T=\varnothing$ and $(X-X) \cap(T-T)=\{0\}$. Since $X$ is inverse-closed, one gets $(X-X) \cap T=\varnothing$, which implies $(-(X-X)) \cap(-T)=\varnothing$. It follows that $(X-X) \cap(-T)=\varnothing$. Since $T^{0}-T^{0}=T \cup(T-T) \cup(-T)$, we have that $(X-X) \cap\left(T^{0}-T^{0}\right)=\{0\}$. Now Lemma 5 implies $A=X \oplus T^{0}$, as desired.

The cyclic group of order $n$ or the additive cyclic group of integers modulo $n$ is denoted by $\mathbb{Z}_{n}=\{0,1, \ldots, n-1\}$. We use the following example to illustrate Corollary 6 .

Example 7. In $\mathbb{Z}_{12}$, let $X=\{0,3,6,9\}$ and $T=\{1,11\}$. Then $(X-X) \cap\left(T^{0}-T^{0}\right)=$ $\{0\}$, and so $\mathbb{Z}_{12}=X \oplus T^{0}$. Therefore, Corollary 6 implies that the Cayley sum graph CayS $\left(\mathbb{Z}_{12}, T\right)$ admits the perfect code $X$.

If CayS $(A, T)$ admits a perfect code, by Theorem 4 we have that $|T|+1$ divides $|A|$. Observe that $2,|A| / 2$ and $|A|$ are divisors of $|A|$ as $|A|$ is even. If $|T|+1=2$, since every 1-regular graph has a perfect code, $\operatorname{CayS}(A, T)$ admits a perfect code. If $|T|+1=|A|$, then $\operatorname{CayS}(A, T)$ is a complete graph, which admits a perfect code of size 1 (in fact, in this case, $A$ is an elementary abelian 2-group, see Corollary 16). Now we consider the case $|T|+1=|A| / 2$.

Corollary 8. A Cayley sum graph $\operatorname{CayS}(A, T)$ of valency $|A| / 2-1$ admits a perfect code if $\left|T-T^{0}\right|<|A|$.

Proof. The condition $\left|T-T^{0}\right|<|A|$ indicates that there exists an element $a \in A$ such that $a \notin T \cup(T-T)$. Let $X=\{0, a\}$. Then $X+X=\{0, a, 2 a\}, X-X=\{0, a,-a\}$. Since $T$ is square-free, we have $(X+X) \cap T=\varnothing$. Since $a \notin T-T$, one gets $-a \notin T-T$. It follows that $(X-X) \cap(T-T)=\{0\}$, and so the desired result follows from Theorem 4 . 
We conclude the section by the following example to illustrate Corollary 8 .

Example 9. Let $n$ be a positive even integer and let $T$ be a square-free subset of $\mathbb{Z}_{n}$. Then $T \subseteq\{1,3, \ldots, n-1\}$. Suppose that $|T|=n / 2-1$. Let $\{a\}=\{1,3, \ldots, n-1\} \backslash T$. Then $a \notin T \cup(T-T)$. Thus, by Corollary 8 , it follows that a Cayley sum graph CayS $\left(\mathbb{Z}_{n}, T\right)$ of valency $n / 2-1$ has a perfect code. As a concrete example, the Cayley sum graph CayS $\left(\mathbb{Z}_{12},\{1,3,5,7,9\}\right)$ admits the perfect code $\{0,11\}$.

\section{$3 \quad$ Subgroup perfect codes}

In this section, we study subgroup perfect codes of an abelian group of even order. In [19], the authors reduced the problem of determining when a given subgroup of an abelian group is a perfect code to the case of abelian 2-groups, and classified the abelian groups whose all non-trivial subgroups are perfect codes. Our main result of this section is Theorem 10, which characterizes the structure of a subgroup perfect code of $A$ and improves [19, Theorem 3.1]. To state our main theorem, we prepare some basic notations.

Let $H$ be a subgroup of $A$. The index of $H$ in $A$, denoted by $[A: H]$, is the number of right (or left) cosets of $H$ in $A$. $H$ is said to be a Hall $2^{\prime}$-subgroup of $A$ if $[A: H]$ is equal to the cardinality of a Sylow 2-subgroup of $A$. We use $A_{2}$ and $A_{2^{\prime}}$ to denote the Sylow 2 -subgroup and Hall $2^{\prime}$-subgroup of $A$, respectively. Note that $A_{2}$ consists of the elements of $A$ each of whose order is a power of 2 , and $A_{2^{\prime}}$ consists of the elements of $A$ with odd order. Particularly, if $A$ is a 2-group, then $A=A_{2}$. As usual, we use $B \times C$ to denote the direct product (or direct sum) of two groups $B$ and $C$. So, we have $A=A_{2} \times A_{2^{\prime}}$. By the fundamental theorem of finitely generated abelian groups, every finite abelian group is a direct product of some cyclic groups. Since $A=A_{2} \times A_{2^{\prime}}$, we may assume that

$$
A=\mathbb{Z}_{2^{m_{1}}} \times \mathbb{Z}_{2^{m_{2}}} \times \cdots \times \mathbb{Z}_{2^{m_{k}}} \times A_{2^{\prime}}
$$

where $m_{i} \geqslant 1$ for each $1 \leqslant i \leqslant k$. Observe that an element $\left(a_{1}, \ldots, a_{k}, a\right)$ in $A$ is not a square if and only if there exists an odd integer in $\left\{a_{1}, \ldots, a_{k}\right\}$. In other words, an element $\left(a_{1}, \ldots, a_{k}, a\right)$ in $A$ is a square if and only if every element of $\left\{a_{1}, \ldots, a_{k}\right\}$ is even. Note that the whole group $A$ is a perfect code in the empty Cayley sum graph $\operatorname{CayS}(A, \varnothing)$.

Theorem 10. Let $A$ be an abelian group as presented in (2), and let $H$ be a subgroup of $A$. Then $H$ is a subgroup perfect code of $A$ if and only if either $H$ is a subgroup isomorphic to

$$
\mathbb{Z}_{2^{m_{1}-1}} \times \mathbb{Z}_{2^{m_{2}-1}} \times \cdots \times \mathbb{Z}_{2^{m_{k}-1}} \times A_{2^{\prime}}
$$

or $H$ has a non-square element.

We use the following example to illustrate Theorem 10.

Example 11. Let $A=\mathbb{Z}_{2} \times \mathbb{Z}_{4} \times \mathbb{Z}_{3}$. Then

$$
H=\{(0,0,0),(0,2,0),(0,2,1),(0,2,2),(0,0,1),(0,0,2)\}
$$


is the subgroup of $A$ which is isomorphic to $\mathbb{Z}_{1} \times \mathbb{Z}_{2} \times \mathbb{Z}_{3}$, and the set of all non-square elements of $A$ is

$$
A \backslash H=\{(1, x, y),(0, z, y): x \in\{0,1,2,3\}, y \in\{0,1,2\}, z \in\{1,3\}\} .
$$

Therefore, Theorem 10 implies that any subgroup perfect code of $A$ is either $H$ or a subgroup $K$ satisfying $K \cap(A \backslash H) \neq \varnothing$. For example, the cyclic subgroup generated by any element belonging to $A \backslash H$ is a subgroup perfect code of $A$.

A right transversal (resp. left transversal) of a subgroup $H$ in $A$ is defined as a subset of $A$ which contains exactly one element in each right coset (resp. left coset) of $H$ in $A$. In an abelian group, every right coset of any subgroup is also a left coset of the subgroup, for the sake of simplicity, we then use the term "transversal" to substitute for "right transversal" or "left transversal".

In order to prove Theorem 10, we need some auxiliary results. Our first result is the following proposition which gives some necessary and sufficient conditions for a subgroup of an abelian group to be a subgroup perfect code of the abelian group. Remark that the following result has its own interest and we only use one equivalent condition in the sequel.

Proposition 12. Let $H$ be a subgroup of $A$. The following are equivalent:

(i) $H$ is a subgroup perfect code of $A$;

(ii) There exists a square-free subset $T \subseteq A$ such that $T^{0}$ is a transversal of $H$ in $A$;

(iii) There exists a square-free subset $T \subseteq A$ such that

$$
[A: H]=|T|+1, \quad H \cap(T \cup(T-T))=\{0\} ;
$$

(iv) There exists a square-free subset $T \subseteq A$ such that $A=H \oplus T^{0}$;

(v) $H$ is a subgroup perfect code of any subgroup of $A$ which contains $H$.

Proof. Combining Theorem 4 and Corollary 6, one can verify that any two of (i), (ii), (iii) and (iv) are equivalent. We next prove that (i) and (v) are equivalent. It is clear that (v) implies (i). Now suppose that $H$ is a subgroup perfect code of $A$. Let $K$ be an arbitrary subgroup of $A$ with $H \subseteq K$. It suffices to prove that $H$ is a subgroup perfect code of $K$. Since (i) and (ii) are equivalent, there exists a square-free subset $T \subseteq A$ such that $T \cup\{0\}$ is a transversal of $H$ in $A$. Let $T^{\prime}=T^{0} \cap K$ where $T^{0}=T \cup\{0\}$. Then $0 \in T^{\prime}$ and $T^{\prime} \backslash\{0\}$ is square-free. Moreover, by the definition of a perfect code, one has

$$
K=A \cap K=\left(H+T^{0}\right) \cap K=H+\left(T^{0} \cap K\right)=H+T^{\prime} .
$$

It follows that $T^{\prime}$ is a transversal of $H$ in $K$. Since (i) and (ii) are equivalent again, $H$ is a subgroup perfect code of $K$. 
Lemma 13. Let $H$ be a subgroup of $A$. If $H$ has a non-square element, then $H$ is a subgroup perfect code of $A$.

Proof. Let $A$ have the form as presented in (2). Suppose that $\left(a_{1}, \ldots, a_{k}, a\right) \in H$ is a non-square element in $A$. Then there exists an odd integer in $\left\{a_{1}, \ldots, a_{k}\right\}$. In fact, without loss of generality, it follows from (2) that we may assume that $a_{1}$ is odd. Now let $\left\{\alpha_{0}, \alpha_{1}, \ldots, \alpha_{s}\right\}$ be a transversal of $H$ in $A$, where $\alpha_{0}$ is the identity element of $A$ and $\alpha_{i}=\left(x_{i 1}, \ldots, x_{i k}, x_{i}\right)$ for each $1 \leqslant i \leqslant s$.

For each $i \in\{1, \ldots, s\}$, if $\alpha_{i}$ is not a square, then let $\beta_{i}=\alpha_{i}$; if $\alpha_{i}$ is a square, then every element of $\left\{x_{j 1}, \ldots, x_{j k}\right\}$ is even, and let

$$
\beta_{i}=\left(a_{1}, \ldots, a_{k}, a\right)+\alpha_{i}=\left(a_{1}+x_{i 1}, \ldots, a_{k}+x_{i k}, a+x_{i}\right)
$$

which imply that $a_{1}+x_{i 1}$ is odd in $\mathbb{Z}_{2^{m_{1}}}$ since $a_{1}$ is odd. It follows that $\beta_{i}$ is a non-square element. Also, note that $\beta_{i} \in H+\alpha_{i}$. We conclude that $\left\{\alpha_{0}, \beta_{1}, \ldots, \beta_{s}\right\}$ is a transversal of $H$ in $A$, and $\left\{\beta_{1}, \ldots, \beta_{s}\right\}$ is square-free in $A$. It follows from Proposition 12 (ii) that $H$ is a subgroup perfect code of $A$.

Denote $A^{\mathrm{II}}=\{2 a: a \in A\}$. Then $A^{\mathrm{II}}$ is the set of all squares of $A$.

Lemma 14. Let $H$ be a subgroup of $A$. Suppose that every element of $H$ is a square. Then $H$ is a subgroup perfect code of $A$ if and only if $H=A^{\mathrm{II}}$.

Proof. Let $A$ have the form as presented in (2). We first prove the necessity. Suppose that $H$ is a subgroup perfect code of $A$. Note that $H \subseteq A^{\mathrm{II}}$. Assume the contrary, namely, there exists an element $\left(a_{1}, \ldots, a_{k}, a\right) \in A^{\mathrm{II}} \backslash H$. Then every element of $\left\{a_{1}, \ldots, a_{k}\right\}$ is even. Also, by Proposition 12 (ii), we have that $H+\left(a_{1}, \ldots, a_{k}, a\right)$ contains at least one non-square element of $A$, a contradiction since the sum of two squares is a square.

We now prove the sufficiency. Suppose that $H=A^{\mathrm{II}}$. Let $\left\{\alpha_{0}, \alpha_{1}, \ldots, \alpha_{s}\right\}$ be a transversal of $H$ in $A$, where $\alpha_{0}$ is the identity element of $A$. It follows that $\left\{\alpha_{1}, \ldots, \alpha_{s}\right\}$ is square-free. Now Proposition 12 (ii) implies that $H$ is a subgroup perfect code of $A$.

We are now ready to prove Theorem 10 .

Proof of Theorem 10. We first claim that $H=A^{\mathrm{II}}$ if and only if $H$ is a subgroup isomorphic to (3). In fact,

$$
\begin{aligned}
A^{\mathrm{II}} & =\left\{2\left(a_{1}, \ldots, a_{k}, a\right):\left(a_{1}, \ldots, a_{k}, a\right) \in A\right\} \\
& =\left\{\left(2 a_{1}, \ldots, 2 a_{k}, 2 a\right):\left(a_{1}, \ldots, a_{k}, a\right) \in A\right\} \\
& =\left\{\left(b_{1}, \ldots, b_{k}, b\right): b_{i} \text { is even and belongs to } \mathbb{Z}_{2^{m_{i}}} \text { for all } 1 \leqslant i \leqslant k, b \in A_{2^{\prime}}\right\} \\
& \cong \mathbb{Z}_{2^{m_{1}-1}} \times \mathbb{Z}_{2^{m_{2}-1}} \times \cdots \times \mathbb{Z}_{2^{m_{k}-1}} \times A_{2^{\prime}} .
\end{aligned}
$$

Thus, the claim is valid. Now combining Lemmas 13 and 14, we complete the proof. 
We next give a proof of [19, Corollary 3.2] using Theorem 10.

Corollary 15. ([19, Corollary 3.2]) An abelian group has a subgroup perfect code of odd order if and only if the Sylow 2-subgroup of the group is an elementary abelian 2-group and the subgroup perfect code is the Hall 2'-subgroup of the group.

Proof. Let $A$ be an abelian group as presented in (2). The proof of the sufficiency is straightforward by Theorem 10. We now prove the necessity. Suppose that $H$ is a subgroup perfect code of $A$ and has odd order. Then $H$ has no non-square elements. Theorem 10 implies that $H$ is a subgroup isomorphic to (3). It follows that $m_{i}=1$ for all $1 \leqslant i \leqslant k$, and so the Sylow 2-subgroup of $A$ is elementary abelian and $H$ is the Hall $2^{\prime}$-subgroup of $A$, as desired.

The next corollary follows immediately from Corollary 15.

Corollary 16. The subgroup consisting of the identity element of an abelian group is a subgroup perfect code if and only if the abelian group is an elementary abelian 2-group.

We next give a necessary condition for a subgroup of an abelian group to be a subgroup perfect code of the abelian group.

Proposition 17. Let $H=H_{2} \times H_{2^{\prime}}$ be a subgroup of $A=A_{2} \times A_{2^{\prime}}$. If $H$ is a subgroup perfect code of $A$, then $H_{2}$ is a subgroup perfect code of $A_{2}$.

Proof. If $H=A$, then $H_{2}=A_{2}$, and so $H_{2}$ is a subgroup perfect code of $A_{2}$ from Theorem 10 , as desired. Thus, in the following, we may assume that $H \neq A$. In view of Proposition 12 (ii), the group $A_{2} \times A_{2^{\prime}}$ has a square-free subset $T:=\left\{\left(x_{1}, y_{1}\right),\left(x_{2}, y_{2}\right), \ldots,\left(x_{s}, y_{s}\right)\right\}$ such that $T \cup\{(0,0)\}$ is a transversal of $H_{2} \times H_{2^{\prime}}$ in $A_{2} \times A_{2^{\prime}}$. Note that $H_{2} \subseteq A_{2}$ and $H_{2^{\prime}} \subseteq A_{2^{\prime}}$. It follows that $\left\{x_{1}, x_{2}, \ldots, x_{s}\right\}$ is a square-free subset of $A_{2}$. Notice that $\bigcup_{i=0}^{s}\left(H_{2}+x_{i}\right)=A_{2}$, where $x_{0}=0$. So, we may assume that $T^{\prime}:=\left\{x_{1}^{\prime}, x_{2}^{\prime}, \ldots, x_{s^{\prime}}^{\prime}\right\}$ is a subset of $\left\{x_{1}, x_{2}, \ldots, x_{s}\right\}$ such that $T^{\prime}$ is square-free and $T^{\prime} \cup\{0\}$ is a transversal of $H_{2}$ in $A_{2}$. Note that (i) and (ii) in Proposition 12 are equivalent. It follows that $H_{2}$ is a subgroup perfect code of $A_{2}$, as desired.

By Corollary 15, it is easy to see that the converse of Proposition 17 is not true.

Finally, as applications of Theorem 10, we determine the subgroup perfect codes of three families of abelian groups. The first result is obtained by applying Theorem 10 to a cyclic group, which determines all subgroup perfect codes of a cyclic group of even order.

Proposition 18. ([19, Theorem 3.7]) Let $A=\mathbb{Z}_{2^{n}} \times \mathbb{Z}_{m}$ be a cyclic group of even order, where $n \geqslant 1$ and $m$ is an odd integer. Then a subgroup of $A$ is a subgroup perfect code if and only if the subgroup is isomorphic to either $\mathbb{Z}_{2^{n-1}} \times \mathbb{Z}_{m}$ or $\mathbb{Z}_{2^{n}} \times \mathbb{Z}_{m^{\prime}}$, where $m^{\prime} \mid m$.

Proposition 19. Let $A=\mathbb{Z}_{2}^{n} \times A_{2^{\prime}}$, where $n \geqslant 1$. Then a subgroup of $A$ is a subgroup perfect code if and only if either the subgroup has even order or the subgroup is $A_{2^{\prime}}$. 
Proof. The necessity follows trivially from Corollary 15. We now prove the sufficiency. By Corollary 15, we only need to prove that if a subgroup of $A$ has even order, then the subgroup is a subgroup perfect code. Now suppose that $H$ is a subgroup of $A$ and has even order. Taking $a \in H$ with order 2 , we have $a \in A_{2}$. Since $A_{2}$ is an elementary abelian 2-group, $A$ has no elements of order 4 , which implies that $a$ is a non-square element. Lemma 13 implies that $H$ is a subgroup perfect code, as desired.

Proposition 20. Let $A=\mathbb{Z}_{2}^{n} \times \mathbb{Z}_{4} \times A_{2^{\prime}}$, where $n \geqslant 1$. Then a subgroup of $A$ is a subgroup perfect code if and only if it is not isomorphic to one of $E_{1}^{n} \times \mathbb{Z}_{2} \times A_{2^{\prime}}^{\prime}$ and $A_{2^{\prime}}^{\prime \prime}$, where $E_{1}$ is the group consisting of the identity element of $\mathbb{Z}_{2}, A_{2^{\prime}}^{\prime}$ is a proper subgroup of $A_{2^{\prime}}$, and $A_{2^{\prime}}^{\prime \prime}$ is a subgroup of $A_{2^{\prime}}$.

Proof. We first prove the necessity. Let $H$ be a subgroup perfect code of $A$. Clearly, by Corollary $15, H$ is not isomorphic to a subgroup of $A_{2^{\prime}}$. Suppose to the contrary that $H \cong E_{1}^{n} \times \mathbb{Z}_{2} \times A_{2^{\prime}}^{\prime}$, where $E_{1}$ is the group consisting of the identity element of $\mathbb{Z}_{2}$ and $A_{2^{\prime}}^{\prime}$ is a proper subgroup of $A_{2^{\prime}}$. Then any element of $H$ has the form

$$
(\underbrace{0, \ldots, 0}_{n}, 0, a) \text { or }(\underbrace{0, \ldots, 0}_{n}, 2, a)
$$

where $a \in A_{2^{\prime}}^{\prime}$. It follows that every element of $H$ is a square. By Theorem 10, it follows that $A_{2^{\prime}}^{\prime}=A_{2^{\prime}}$, which contradicts that $A_{2^{\prime}}^{\prime}$ is a proper subgroup of $A_{2^{\prime}}$.

We next prove the sufficiency. Suppose that a subgroup $H$ of $A$ is not isomorphic to one of $E_{1}^{n} \times \mathbb{Z}_{2} \times A_{2^{\prime}}^{\prime}$ and $A_{2^{\prime}}^{\prime \prime}$. If $H \cong E_{1}^{n} \times \mathbb{Z}_{2} \times A_{2^{\prime}}$, then Theorem 10 implies that $H$ is a subgroup perfect code, as desired. Now suppose that $H \nsubseteq E_{1}^{n} \times \mathbb{Z}_{2} \times A_{2^{\prime}}$. Then by the structure of $A$, we conclude that $H$ has a non-square element, and so $H$ is a subgroup perfect code by Lemma 13, as required.

\section{Acknowledgements}

The authors thank the referee for thorough comments that improve the presentation of this paper.

\section{References}

[1] N. Alon. Large sets in finite fields are sumsets. J. Number Theory, 126:110-118, 2007.

[2] N.L. Biggs. Perfect codes in graphs. J. Combin. Theory Ser. B, 15:289-296, 1973.

[3] J. Chen, Y. Wang, B. Xia. Characterization of subgroup perfect codes in Cayley graphs. Discrete Math., 343:111813, 2020.

[4] B. Cheyne, V. Gupta, C. Wheeler. Hamilton cycles in addition graphs. Rose-Hulman Undergrad. Math J., 4:1-17, 2003.

[5] F.R.K. Chung. Diameters and eigenvalues. J. Amer. Math. Soc., 2:187-196, 1989. 
[6] I.J. Dejter, O. Serra. Efficient dominating sets in Cayley graphs. Discrete Appl. Math., 129:319-328, 2003.

[7] P. Delsarte. An algebraic approach to the association schemes of coding theory. Philips Res. Rep. Suppl., 10, 1973.

[8] Y.-P. Deng. Efficient dominating sets in circulant graphs with domination number prime. Inform. Process. Lett., 114:700-702, 2014.

[9] Y.-P. Deng, Y.-Q. Sun, Q. Liu, H.-C. Wang. Efficient dominating sets in circulant graphs. Discrete Math., 340:1503-1507, 2017.

[10] M. DeVos, L. Goddyn, B. Mohar, R. Sámal. Cayley sum graphs and eigenvalues of (3,6)-fullerenes. J. Combin. Theory Ser. B, 99:358-369, 2009.

[11] R. Feng, H. Huang, S. Zhou. Perfect codes in circulant graphs. Discrete Math., 340:1522-1527, 2017.

[12] D. Grynkiewicz, V.F. Levb, O. Serra. Connectivity of addition Cayley graphs. J. Combin. Theory Ser. B, 99:202-217, 2009.

[13] O. Heden. A survey of perfect codes. Adv. Math. Commun., 2:223-247, 2008.

[14] H. Huang, B. Xia, S. Zhou. Perfect codes in Cayley graphs. SIAM J. Discrete Math., 32:548-559, 2018.

[15] S.V. Konyagin, I.D. Shkredov. On subgraphs of random Cayley sum graphs. European J. Combin., 70:61-74, 2018.

[16] J. Kratochvíl. Perfect codes over graphs. J. Combin. Theory Ser. B, 40:224-228, 1986.

[17] J. Lee. Independent perfect domination sets in Cayley graphs. J. Graph Theory, $37: 213-219,2001$.

[18] V.F. Lev. Sums and differences along Hamiltonian cycles. Discrete Math., 310:575$584,2010$.

[19] X. Ma, M. Feng, K. Wang. Subgroup perfect codes in Cayley sum graphs. Des. Codes Cryptogr., 88:1447-1461, 2020.

[20] X. Ma, G.L. Walls, K. Wang, S. Zhou. Subgroup perfect codes in Cayley graphs. SIAM J. Discrete Math., 34:1909-1921, 2020.

[21] X. Ma, K. Wang. Integral Cayley sum graphs and groups. Discuss. Math. Graph Theory, 36:797-803, 2016.

[22] M. Mollard. On perfect codes in Cartesian products of graphs. European J. Combin., 32:398-403, 2011.

[23] J.H. van Lint. A survey of perfect codes. Rocky Mountain J. Math., 5:199-224, 1975.

[24] D.T. Vuza. Supplementary sets and regular complementary unending canons (part one). Perspect. New Music, 29:22-49, 1991.

[25] J. Zhang, S. Zhou. On subgroup perfect codes in Cayley graphs. European J. Combin., 91:103228, 2021. 
[26] J. Zhang, S. Zhou. Corrigendum to "On subgroup perfect codes in Cayley graphs" [European J. Combin. 91 (2021) 103228]. European J. Combin., 103461, 2021. In Press, https://doi.org/10.1016/j.ejc.2021.103461.

[27] J. Žerovnik. Perfect codes in direct products of cycles - a complete characterization. Adv. Appl. Math., 41:197-205, 2008.

[28] S. Zhou. Total perfect codes in Cayley graphs. Des. Codes Cryptogr., 81:489-504, 2016.

[29] S. Zhou. Cyclotomic graphs and perfect codes. J. Pure Appl. Algebra, 223:931-947, 2019. 